\title{
El fomento del empleo decente y sostenible en cooperativas y sociedades laborales
}

\author{
Sergio Canalda Criado ${ }^{1}$
}

Recibido: 28 de febrero de 2019 / Aceptado: 4 de julio de 2019

Resumen. Los poderes públicos, a través del fomento de la economía social y la persecución del pleno empleo, deben promover la creación de empleo estable y de calidad. En dicho marco deben situarse las subvenciones a la incorporación de personas socias trabajadoras o de trabajo en cooperativas y sociedades laborales. La creación de empleo como consecuencia de la intervención de los poderes públicos a través del fomento de éste se puede estudiar cuantitativamente, tomando en cuenta el número de puestos de trabajo que se generan a través de las políticas de empleo. Sin embargo, lo que aquí se propone es un estudio del empleo en términos cualitativos, esto es, el tipo de empleo generado, tomando en cuenta las entidades beneficiarias, los colectivos cuya incorporación se subvenciona y los criterios de selección y obligaciones asumidas por las sociedades con la concesión de la subvención.

Palabras clave: Economía Social; Cooperativas; Sociedades Laborales; Fomento de Empleo Digno.

Claves Econlit: J54; P13; L31; J21.

\section{[en] The promotion of decent and sustainable employment in cooperatives and labor societies}

\begin{abstract}
The promotion of the social economy and the pursuit of full employment by public authorities should promote the creation of stable and quality employment. In this context, employment policies must be targeted at the incorporation of members in cooperatives and workerowned companies. The creation of employment as a result of the intervention of the public powers can be assessed quantitatively, taking into account the number of jobs created through employment policies. However, this paper focuses on the type of employment in qualitative terms, studying the beneficiary entities, the social groups whose access to membership is granted, and the selection criteria and the obligations assumed by beneficiaries.
\end{abstract}

Keywords: Social Economy; Cooperatives; Labor Societies; Promotion of Decent Employment.

Sumario. 1. La economía social y la persecución del pleno empleo: hacia el empleo decente y sostenible. 2. El fomento del empleo en la economía social: especial atención a cooperativas y sociedades laborales. 3. Análisis del empleo fomentado en cooperativas y sociedades laborales a través de las ayudas para la incorporación de nuevas personas socias trabajadoras y de trabajo. 4. Conclusiones: el impulso del empleo estable y de calidad a través de nuevos criterios y obligaciones. 5. Referencias bibliográficas. 6. Referencias legales.

1 Universitat Pompeu Fabra, España

Dirección de correo electrónico: sergio.canalda@upf.edu 
Cómo citar: Canalda Criado, S. (2019) El fomento del empleo decente y sostenible en cooperativas y sociedades laborales. REVESCO. Revista de Estudios Cooperativos, Tercer Cuatrimestre, $\mathrm{N}^{\circ} 132$, pp. 77-96. DOI: 10.5209/REVE.65485.

\section{La economía social y la persecución del pleno empleo: hacia el empleo decente y sostenible}

Con motivo de su centenario, la Organización Internacional del Trabajo (OIT) puso en marcha una Comisión Mundial sobre el Futuro del Trabajo, la cual ha emitido en 2019 el informe "Trabajar para un futuro más prometedor" en dónde se recogen los tres ejes de un programa de trabajo sobre los que debiera operar la actuación de los Estados y el cuál se concibe centrado en las personas. Uno de esos tres ejes es incrementar la inversión en trabajo decente y sostenible, siendo una de las dos propuestas concretas en esta materia transformar las economías para promover el trabajo decente y sostenible y, de acuerdo con ello, la Comisión exhorta "a la adopción de incentivos para promover las inversiones en áreas clave que promuevan el trabajo decente y sostenible" (Oficina Internacional del Trabajo, 2019: 49). En esta senda se sitúa el fomento de la economía social, pues ésta se caracteriza por priorizar las personas y el fin social sobre el capital y los beneficios económicos.

El empleo calificado como 'decente' -también denominado como 'empleo digno' - y 'sostenible' hace referencia a aquél que se caracteriza por disfrutar de los estándares sociales que recoge el programa de Trabajo Decente, incorporado a la acción de la OIT en la Declaración sobre la justicia social para una globalización equitativa de 2008 y producirse en empresas sostenibles. Partiendo de ello, el análisis que se presenta a continuación pretende responder a la pregunta de si las políticas de fomento del empleo en sociedades cooperativas y sociedades laborales son coherentes con el impulso de un empleo que pueda ser calificado como tal. De acuerdo con ello, la hipótesis que se plantea es que las subvenciones para la incorporación de nuevas personas socias trabajadoras y de trabajo en cooperativas y sociedades laborales desarrolladas en las CC.AA.: (1) estimulan la creación de empleo en sectores de actividad y en ocupaciones con una proyección de futuro favorable al mantenimiento del empleo: (2) atienden a las necesidades de especial empleabilidad de colectivos específicos a la hora de regular las personas que pasan a adquirir la condición de socias gracias a dicha subvención; y (3) incluyen entre los criterios para seleccionar a las sociedades beneficiarias, así como las obligaciones asumidas con la subvención, unas condiciones de empleo que, a su vez, favorezcan el empleo decente y sostenible.

La aportación científica que aquí se pretende realizar es el análisis jurídico de las ayudas para la incorporación de nuevas personas socias trabajadoras y de trabajo, ateniendo a la dimensión cualitativa del empleo y complementando así los estudios cuantitativos que han revelado, por ejemplo, el impacto del empleo generado en términos comparativos con el empleo total en estas sociedades (Chaves Ávila y Savall Morera, 2013). Además, el trabajo realizado cohonesta los trabajos que constatan el hecho de que las sociedades cooperativas y las sociedades laborales se caracterizan en términos generales por mantener un empleo de calidad (Calderón y Calderón, 2012) y, por otro lado, lo apuntado por Clemente et al 
(2009: 66), quienes señalaron la necesidad de analizar la influencia de las diferentes políticas de las CC.AA. en materia de empresas cooperativas y sociedades laborales para comprender la divergencia, por ejemplo, en cuanto al empleo.

Desde esta forma, el estudio del fomento del empleo en las entidades de la economía social por parte de los poderes públicos en España debe partir, por un lado, del deber de persecución del pleno empleo, de acuerdo con con la propia configuración de éste en nuestro orden constitucional y, por otro lado, de las obligaciones del Estado según los compromisos adquiridos a nivel internacional y europeo, en cuanto a su responsabilidad de fomentar un empleo estable y digno.

Respecto a los compromisos internacionales, la Recomendación núm. 193 de la OIT sobre la promoción de las cooperativas reconoce la directa conexión entre éstas y el empleo decente y sostenible. Así, señala el papel de los gobiernos relativo a la promoción del empleo o el desarrollo de actividades en beneficio de los grupos o regiones desfavorecidas y la especial atención que debe prestarse al incremento de la participación de las mujeres en el movimiento cooperativo. En cuanto a las políticas a implementar, la Recomendación establece el deber de "promover la adopción de medidas para garantizar que se apliquen las mejores prácticas laborales" (apartado $8^{\circ}$ ). En el ámbito de la Unión Europea, la Resolución del Parlamento Europeo de 19 de febrero de 2009, sobre economía social (Parlamento Europeo, 2008), ha afirmado que "la economía social, mediante la conjunción de rentabilidad y solidaridad, desempeña un papel esencial en la economía europea al permitir la creación de empleos de calidad" y que ésta "tiene un papel clave que desempeñar en el logro de los objetivos de la Estrategia de Lisboa relativos al crecimiento sostenible y al pleno empleo". La Estrategia Europea 2020 plantea entre sus prioridades un crecimiento económico integrador basado en el "fomento de una economía con alto nivel de empleo que tenga cohesión social y territorial" (Comisión Europea, 2010). En relación a ello, dentro del ámbito de aplicación del Fondo Social Europeo se incluye el "fomento del emprendimiento social y de la integración profesional en las empresas sociales, así como de la economía social y solidaria, a fin de facilitar el acceso al empleo" (art. 3.1.b.v. del Reglamento (UE) n ${ }^{\circ}$ 1304/2013, de 17 de diciembre de 2013).

En el ordenamiento jurídico español, la Constitución Española (CE) reconoce el deber de los poderes públicos de promover las condiciones favorables para el progreso social y económico y de realizar una política orientada al pleno empleo (art. 40 CE). Como ha afirmado el Tribunal Constitucional, el art. 40 CE recoge la vertiente colectiva del derecho al trabajo, mientras que su vertiente individual se consagra en el art. 35 CE (Sentencia del Tribunal Constitucional de 2 julio 1981, cuestión de inconstitucionalidad 223/1981). Por otro lado, los poderes públicos deberán fomentar "mediante una legislación adecuada, las sociedades cooperativas" así como establecer los medios "que faciliten el acceso de los trabajadores a la propiedad de los medios de producción" (art. 129.2 CE). En relación a éste último, se ha afirmado que la denominada 'Constitución Económica' configura la participación en la empresa "desde una concepción comunitaria de la misma y una verdadera promoción de la economía social y solidaria en la que se otorga un especial relieve a las cooperativas" (Colomer Viadel, 2017: 835). Montoya Melgar (2004: 27-28) ha afirmado que la 
participación en la empresa sería un principio rector de la vida económica y social fuera del capítulo III del Título I ("De los principios rectores de la política social y económica") siendo poco explicable que se ubiquen en el Título VII ("Economía y Hacienda") debido a su contenido eminentemente social. Sobre ello, Nogueira Guastavino (2018:1800) ha afirmado que su ubicación en el texto Constitucional responde a la voluntad de "agrupar los mecanismos posibles de intervención en la economía por parte de los poderes públicos”. También, Paniagua Zurera y Jiménez Escobar (2014: 64) afirman que la razón para su ubicación radica en "el fuerte o mayoritario contenido económico que preside el mandato de fomento hacia las sociedades cooperativas - y, hoy, por extensión, a las restantes empresas y entidades de la economía social".

La interrelación de ambos preceptos (40 CE y $129 \mathrm{CE}$ ) es indudable por dos razones. En primer lugar, porque ambos pivotan sobre la configuración del "Estado Social" (art. 1.1 CE) y sobre la cláusula de 'igualdad real' que manda a los poderes públicos "promover las condiciones para que la libertad y la igualdad del individuo y de los grupos en que se integra sean reales y efectivas; remover los obstáculos que impidan o dificulten su plenitud y facilitar la participación de todos los ciudadanos en la vida política, económica, cultural y social". Y, en segundo lugar, porque la consecución de ambos deriva en el mismo fin: mientras que las medidas de fomento del empleo "deben ir dirigidas a generar las condiciones que permitan a todo aquel que lo requiera optar a un empleo estable y de calidad" (Cavas Martínez, 2016a: 54), las cooperativas, en el caso de trabajo asociado, ofrecen la posibilidad de dar efectividad a al derecho al trabajo (art. $35 \mathrm{CE}$ ) y la propia consecución del pleno empleo (art. 40 CE) (Díaz de la Rosa, 2010: 320). También podríamos llegar a la misma conclusión respecto de las sociedades laborales pues, como afirma Del Pino Domínguez (2016: 12), del principio orientador de la primacía de las personas y del fin social sobre el capital se extrae el requisito por el cual en dichas sociedades la mayoría del capital social deber ser propiedad de trabajadores de la empresa con una relación laboral por tiempo indefinido.

La evolución de las políticas de empleo y del fomento de la economía social se hace indisociable atendiendo al hecho de que las medidas de fomento del empleo reservadas tradicionalmente a la contratación de trabajadores en régimen laboral o asalariado se han ido extendiendo al autoempleo en entidades de economía social (Cavas Martínez, 2016b). Así, es relevante centrar el estudio en las cooperativas de trabajo asociado y las sociedades laborales, por concebirse como fórmulas del "autoempleo colectivo" (Bengoetxea Alkorta, 2016:15). A continuación, se presentará el marco legal desde donde parte las políticas de fomento del empleo en cooperativas y sociedades laborales (apartado $2^{\circ}$ ) para después realizar el estudio del tipo de empleo fomentado a través de las políticas implementadas en el ámbito autonómico desde un punto de vista cualitativo (apartado $3^{\circ}$ ).

\section{El fomento del empleo en la economía social: especial atención a cooperativas y sociedades laborales}

Las crisis económicas de 2008 y de 2010 dejaron tras de sí la mayor destrucción de empleo de las últimas décadas. Sin embargo, durante la primera fase de la crisis las 
cooperativas y las sociedades laborales resistieron más que las empresas del sector privado en términos de empleo (Sala, et al, 2014: 25). Esta resistencia se podría justificar, entre otras causas, porque el Derecho cooperativo únicamente contempla la "baja obligatoria (despido) cuando la situación sea de tal entidad, de tal gravedad, que sea una medida adecuada para mantener la viabilidad económica de la cooperativa" (Bengoetxea Alkorta, 2015: 46). Con todo, si bien durante la segunda crisis hubo también destrucción neta de empleo en aquellos tipos de sociedades, Sala et al. (2014: 25) insisten en potenciar dichas sociedades por su capacidad de generar empleo antes de que comience la fase de recuperación y, durante las crisis, ofrecer las sociedades laborales un elevado grado de resistencia y las cooperativas, por su parte, una menor pérdida de empleo en términos relativos.

En este contexto de crisis y el grave problema social que dicha situación provocó, el gobierno aprobó la Ley 5/2011, de 29 de marzo, de Economía Social (LES) motivado, entre otras razones, por la resistencia más potente que mostraron las empresas de economía social en términos de empleo. Por lo tanto, aunque pueden señalarse diferentes factores que impulsaron el debate en relación a la aprobación de la norma (Alfonso Sánchez, 2009), ésta se contextualiza inevitablemente en el cambio de modelo económico que se pretendía a través de la Ley $2 / 2011$, de 4 de marzo, de Economía Sostenible y, especialmente, en la capacidad de las entidades de la economía social para generar empleo estable, como se puede comprobar de la intervención de los participantes en la sesión de la Comisión de Trabajo e Inmigración en la cual se debatió el proyecto de ley de economía social (Diario de Sesiones del Congreso de los Diputados núm. 696 de 21 diciembre de 2010: 2). La modificación de la LES por la Ley 31/2015 corrobora esta afirmación pues, de acuerdo con De Kostka Fernández Fernández (2015: 403), ésta última persiguió fomentar el empleo en estas entidades ya que "tras la crisis, está dando muestras de una alta capacidad de recuperación y un alto potencial de creación de empleo".

De esta forma, la Ley de Economía Social finalmente aprobada tiene por objeto "establecer un marco jurídico común para el conjunto de entidades que integran la economía social (...) así como determinar las medidas de fomento a favor de las mismas en consideración a los fines y principios que les son propios" (art. 1 LES). La interrelación entre la identificación de las entidades de economía social y las medidas de fomento ha sido destacada por Arrieta (2014: 34), citando a Fajardo (2012), afirmando que con la delimitación de cuáles son las entidades que desarrollan su actividad en el campo de la economía social, se busca también "encauzar adecuadamente" las medidas de fomento de la economía social.

El fomento de la economía social es, por tanto, uno de los fines que persigue la norma. Hay que tener en cuenta que, como ha afirmado Fajardo (2012: 94), el fomento de la economía social se ha generalizado en España a todas las Administraciones, si bien "el grado de implicación de las políticas públicas en dicho fomento, es dispar y dependiente de cada Comunidad Autónoma". Así, el marco regulador de dicho fomento que fija la LES en su artículo 8 parte del reconocimiento de éste como tarea de interés general y que los poderes públicos, en el ámbito de sus respectivas competencias, tendrán como objetivo de sus políticas la promoción de la economía social. De entre las políticas de fomento de la economía social, la LES incluye "[i]nvolucrar a las entidades de la economía social 
en las políticas activas de empleo, especialmente en favor de los sectores más afectados por el desempleo, mujeres, jóvenes y parados de larga duración" (art. 8.2.g. LES). Tras la modificación de la norma por la Ley 31/2015, de 9 de septiembre, se incorporaron nuevas medidas que fomentaban expresamente la incorporación de trabajadores a las entidades de la economía social.

Aquella reforma se presentó como un impulso en la legislación de "la materia en autoempleo individual colectivo, con la unívoca pretensión de crear empleo estable, de calidad y en colectivos con mayores dificultades" (De Kostka Fernández Fernández, 2015: 403). Como se puede apreciar, las medidas de fomento de la economía social coincide con las políticas activas de empleo: la Ley de empleo (LE) cuyo Texto Refundido se aprueba por Real Decreto Legislativo $3 / 2015$, de 23 de octubre, define las políticas activas de empleo como un instrumento de la política de empleo consistente en "el conjunto de servicios y programas de orientación, empleo y formación profesional para el empleo en el ámbito laboral dirigidas a mejorar las posibilidades de acceso al empleo, por cuenta ajena o propia, de las personas desempleadas, al mantenimiento del empleo y a la promoción profesional de las personas ocupadas y al fomento del espíritu empresarial y de la economía social" (art. 36.1 LE).

La Estrategia Española de Activación para el Empleo (EEAE), aprobado por Real Decreto 1032/2017, de 15 de diciembre, es el instrumento de coordinación del Sistema Nacional de Empleo que incluye los principios de actuación y objetivos estratégicos y estructurales a alcanzar en materia de políticas de activación para el empleo, "incluyendo políticas activas de empleo y de intermediación laboral, para el conjunto del Estado" (art. 10.2.b. LE). Dentro de la EEAE se incluyen las actividades dirigidas a fomentar la iniciativa empresarial, el trabajo autónomo y la economía social y así queda plasmado en la vigente estrategia para el periodo 2017-2020. De entre los programas y servicios que se incluyen en los Planes Anuales de Política de Empleo (PAE) se encuentran las subvenciones para el fomento del empleo que regula la Orden TAS/3501/2005, de 7 de noviembre, por la que se establecen las bases reguladoras para la concesión de subvenciones para el fomento del empleo y mejora de la competitividad en las cooperativas y sociedades laborales. Dichos incentivos también se prevén entre los ejes de actuación aprobados en la Estrategia Española de la Economía Social (EEES) 2017-2020 (Resolución de 15 de marzo de 2018, de la Secretaría de Estado de Empleo, por la que se publica el Acuerdo del Consejo de Ministros de 29 de diciembre de 2017, por el que se aprueba la Estrategia Española de Economía Social 2017-2020).

Concretamente, la Orden TAS/3501/2005 incluye el programa dirigido a fomentar "la incorporación, con carácter indefinido, como socios trabajadores o de trabajo en cooperativas y sociedades laborales de desempleados y de trabajadores con contrato de trabajo de carácter temporal en la misma cooperativa o sociedad laboral en la que se integran como socios" (art. 1 Orden TAS/3501/2005). Ahora bien, el interés constitucional perseguido no queda satisfecho por la simple incorporación de las personas socias trabajadoras y de trabajo, razón por la cual la propia Orden así como la regulación de las subvenciones en las diferentes Comunidades Autónomas incluyen otros criterios para favorecer que el empleo fomentado sea realmente estable y de calidad. 
Como se avanzaba anteriormente, el estudio que se propone a continuación consiste en un análisis de las diferentes políticas activas de empleo en relación al fomento de la incorporación de nuevas personas socias en cooperativas y sociedades laborales, implementadas a nivel autonómico, con especial atención a los requisitos legales que, previstos para su concesión, pueden favorecer la generación de empleo decente y sostenible.

\section{Análisis del empleo fomentado en cooperativas y sociedades laborales a través de las ayudas para la incorporación de nuevas personas socias trabajadoras y de trabajo}

Antes de entrar a analizar el objeto de estudio, es pertinente hacer una distinción en cuanto al tipo jurídico de 'empleo' generado por cuanto que, por un lado, en las sociedades laborales los socios-propietarios conservan también su cualidad de trabajadores asalariados, en las cooperativas de trabajo asociado, por otro lado, los socios trabajadores carecen de la condición de asalariados y su relación es societaria. En relación a la aplicación de las normas laborales a los socios trabajadores de cooperativas, los poderes legislativos han procedido a una asimilación parcial, siendo lo más importante que, como ha afirmado Trujillo Díez (2002: 407), la razón para dicha asimilación se encuentra fuera de los principios tuitivos propios del Derecho del Trabajo. Es más, según García Jiménez (205: 106), la calificación como societaria de la relación y la desregulación de todo aquello relativo a la prestación de los socios trabajadores, ha dejado el trabajo de éstos en "una especie de limbo, en todas aquellas situaciones de las que la legislación laboral no se ha ocupado expresamente".

Partiendo de esta premisa, cobra aún más importancia el análisis cualitativo del empleo fomentado en las cooperativas, pero también en las sociedades laborales ya que las políticas de las empresas en relación a los trabajadores pueden tenerse en cuenta en el procedimiento de la concesión de la subvención. Por ello, a continuación se estudiarán aquellos elementos de los programas de fomento del empleo en ambos tipos de sociedades que pueden tener un impacto en la creación de un empleo estable y de calidad. Por un lado, las cooperativas y sociedades laborales que pueden beneficiarse de la ayuda y bajo qué requisitos, tanto desde el punto de vista del tamaño así como del sector de actividad y, en relación a ello, si las políticas de empleo se concentran en un tipo de sector relacionado con los nuevos yacimientos de empleo y las actividades económicas y ocupaciones con mejores perspectivas. Por otro lado, se debe estudiar los colectivos de personas trabajadoras que se benefician de dichas políticas, con especial atención a si pertenecen a colectivos que merecen especial atención de acuerdo con la Estrategia Española de Activación para el Empleo. Y finalmente, es necesario prestar también atención a las obligaciones adquiridas por las entidades beneficiarias como, por ejemplo, sobre mantenimiento del empleo o respecto del tipo de jornada. 


\subsection{El destinatario de la subvención: ¿el foco sobre los nuevos yacimientos de empleo?}

La delimitación de las entidades posibles beneficiarias atendiendo a sus características más allá de la forma societaria puede tener un beneficio en cuanto al incentivo del empleo en determinados sectores y tejido productivo. En este sentido, como más adelante se mencionará, entre los criterios que las administraciones deben aplicar para otorgar las ayudas se encuentran algunos parámetros como la relación con los nuevos yacimientos de empleo definidos por la Unión Europea, la incorporación de nuevas tecnologías de la información y las comunicaciones, el desarrollo de proyectos de $\mathrm{I}+\mathrm{D}+\mathrm{i}$ o la incidencia en la creación y mantenimiento del empleo en el entorno geográfico. Por lo tanto, el legislador es consciente de que hay determinadas actividades empresariales que deben ser objeto de especial atención y, por ello, sería posible realizar distinciones en cuanto a las entidades beneficiarias que pudieran concurrir al proceso, en función del propósito del legislador.

La primera referencia a tener en cuenta es, por lo tanto, lo establecido en el artículo 4 de la Orden según el cual podrán ser beneficiarias de las subvenciones para la actuación de incorporación de socios trabajadores o socios de trabajo las cooperativas y sociedades laborales que estén legalmente constituidas, que estén al corriente de las obligaciones tributarias y de Seguridad Social y, por último, no hayan sido sancionadas mediante resolución firme con la pérdida de la posibilidad de obtener subvenciones.

El mismo artículo introduce el sector como factor excluyente para poder ser entidad beneficiaria en el apartado $3^{\circ}$, según el cual las empresas pertenecientes al sector del transporte, aquellas que desarrollen actividades relacionadas con "la producción, primera transformación o comercialización al por mayor de los productos que figuran en el anexo I del Tratado Constitutivo de la Comunidad Europea" y actividades de exportación. Es decir, el único criterio que se introduce en relación al sector está justificado por la política común de agricultura y pesca.

A partir de aquí, la segunda referencia se encuentra en las posibles modificaciones introducidas por las diferentes regulaciones a nivel autonómico que han variado el amplio espectro de empresas que podrían verse beneficiadas de estas subvenciones en al menos dos aspectos: el tamaño de la empresa que puede obtener la subvención y el sector de actividad.

En cuanto al sector de actividad, la norma autonómica ha introducido más exclusiones. Por ejemplo, la norma de Castilla-La Mancha introduce otros sectores excluidos como son vivienda e inmobiliarias, seguros y crédito. En el caso de Extremadura, se prevé, respecto de las cooperativas, la inclusión de éstas que tengan la condición "tanto ordinarias como especiales, calificadas como tales e inscritas en el Registro de Sociedades Cooperativas de Extremadura, excepto las cooperativas de crédito y las de seguros" $\mathrm{y}$, en general, las sociedades cooperativas y sociedades laborales que "deriven de una asociación sin ánimo de lucro" (art. 3 del Decreto 89/2016). También la norma de la Región de Murcia prevé específicamente los sectores de actividad como la pesca y acuicultura, del carbón, etc. Tanto Murcia como Extremadura excluyen aquellas sociedades cooperativas y 
laborales cuando la actividad económica desarrollada corresponda con las propias de las empresas de trabajo temporal o de agencias de colocación.

En cuanto al tamaño de la empresa, podemos encontrar regulación autonómica que ciñe a las pequeñas y medianas empresas el ámbito de aplicación de la subvención. Así, en Castilla-La Mancha, la norma concretiza "[l]os efectivos y límites financieros que definen a la categoría de pequeñas y medianas empresas" (art. 4 del Decreto 22/2017). También es el caso de Murcia, en dónde se limita a las pequeñas y medianas empresas de Economía Social, y se añade una segunda criba al exceptuar aquellas que "[e]stén participadas por otras empresas privadas en cuantía superior al 25\% del capital social, excepto que las empresas participantes sean de Economía Social o sean Organizaciones Empresariales de Economía Social" (art. 4.2.a. de la Orden de 2 de mayo de 2016).

Por último, otro de los requisitos que aquí simplemente se quiere destacar por su relación con los criterios que después deberán utilizarse para resolver la concesión de las subvenciones y que está interrelacionado con el empleo que se genera es el de la territorialidad, entendido éste como aquél que exige que el centro de trabajo dónde se genere el empleo esté ubicado en la CCAA que concede la subvención. Así, por ejemplo, la norma en Aragón exige "[t]ener el centro de trabajo en el que se genera el empleo subvencionado" en algún municipio de la Comunidad Autónoma (art. 3.2.b. de la Orden EIE/607/2016, de 6 de junio). En el mismo sentido podemos citar las normas de la Región de Murcia, de Galicia, Navarra, y País Vasco. En el caso de Cataluña, se prevé el requisito de tener un centro de trabajo en Cataluña (Base 3.1.o. de las Bases Reguladoras incluidas en el Anexo de la Orden TSF/188/2016, de 27 de junio), con independencia del lugar de la sede del domicilio social o del registro dónde esté inscrita. En cambio, Asturias sólo especifica que las cooperativas y las sociedades laborales deberán estar radicadas en el territorio del Principado (Base 3.a. de las Bases reguladoras recogidas en el Anexo I de la Resolución de 1 de agosto de 2013), mientras que la de Castilla y León (Orden de 22 de diciembre de 2017) añade "con domicilio social en Castilla y León y que tengan ánimo de lucro".

Podemos concluir, por lo tanto, que la regulación autonómica perfila las posibles entidades beneficiarias en términos de actividad económica, tamaño de la empresa y su vinculación al territorio. Ahora bien, el contenido de la normativa no parece favorecer la generación de empleo en relación a algunos de los criterios de ponderación mencionados. Aunque si bien es cierto que los criterios son de aplicación posterior durante el procedimiento, hubiera sido deseable orientar el establecimiento de los requisitos de las entidades hacia los criterios que después se valorarán. Por ejemplo, para conseguir una diversificación de la distribución sectorial y, más concretamente, impulsar los nuevos yacimientos de empleo. Éstos son, según San Martín Mazzucconi (2010: 89), los "campos de actividad novedosos (bien por su previa inexistencia, bien por su escaso desarrollo) que se perfilan como puertas de acceso al mercado de trabajo, abiertas en virtud de las necesidades de una sociedad moderna".

En este sentido, cabe preguntarse si no hubiese sido recomendable restringir la participación a las cooperativas y sociedades laborales que desarrollaran una actividad en dónde se detectara una proyección favorable en cuanto a empleo. A modo de ejemplo, la normativa podría restringir la participación a entidades de 
alguna de las actividades económicas cuyas ocupaciones muestren mejores perspectivas entre las cuales se podría señalar, de acuerdo con la EEAE 2017-2020, la enseñanza, las ingenierías en general y las profesionales TIC. De la misma forma, se podrían tener en cuenta los Estudios prospectivos sectoriales que elabora el Servicio Público de Empleo y que presentan las ocupaciones con mejores perspectivas de empleo en sectores como el Comercio Minorista, el de Bienes de Equipo y el de Servicios Avanzados a las Empresas en las Tecnologías de la Información y Comunicación (TIC).

\subsection{Los colectivos cuya incorporación se subvenciona: la ampliación de supuestos}

Qué duda cabe que la acción de los poderes públicos a través de este tipo de subvenciones no debe sólo focalizarse en la promoción del empleo a través de un tipo de agente económico sino también a través del fomento del empleo de colectivos que estén infraocupados en un sector específico o que muestren dificultades en su empleabilidad. Este objetivo está también contemplado en la Orden estatal y la normativa autonómica correspondiente, favoreciendo el empleo de los colectivos menos representados. Ahora bien, la pregunta que deberíamos responder es si los poderes públicos desincentivan otras características del empleo como, por ejemplo, una alta tasa de parcialidad en el empleo femenino.

La EEES 2017-2020 establece cuáles son las características del empleo en las sociedades laborales y en las cooperativas, de cuyo análisis podrían deducirse cuáles serían las características del empleo a fomentar. Así, la EEES califica al empleo femenino como limitado en las sociedades laborales pero en cambio representa el 47,1 \% del empleo en las cooperativas; en lo relativo al empleo juvenil, éste sería adecuado en tanto que representa el $42 \%$ del total de trabajadores tiene menos de 40 años aunque incidiría especialmente en el sector de la agricultura; en relación al empleo a tiempo parcial, éste representaría 19,9\% del total de trabajadores, aunque aumentaría en las sociedades laborales limitadas $(26,6$ $\%)$ y aún más con relación a las mujeres (31,8\%); finalmente, la temporalidad sería positivamente reducida en cooperativas $(23,2 \%)$ pero más elevada en sociedades laborales limitadas $(35,8 \%)$.

A continuación se analizará las previsiones de la Orden estatal en cuanto a los colectivos específicos incluidos para, a continuación, detener el análisis en el ámbito autonómico.

La Orden menciona dos colectivos cuya incorporación con carácter indefinido como socios trabajadores o de trabajo en cooperativas y sociedades laborales son objeto de subvención: uno formado por desempleados, en el cual se incluyen diferentes colectivos, y otro de trabajadores con contrato de trabajo de carácter temporal en la misma cooperativa o sociedad laboral en la que se integran como socios.

Respecto del primer colectivo, la Orden prevé en el art. 3.1 que los desempleados que se incorporen deberán estar incluidos en alguno de los colectivos siguientes: (a) Desempleados menores de 25 años que no hayan tenido antes un primer empleo fijo; (b) Desempleados mayores de 45 años; (c) Desempleados de larga duración que hayan estado sin trabajo e inscritos en la 
oficina de empleo durante al menos 12 de los anteriores 16 meses o durante 6 meses de los anteriores 8 meses si fueran menores de 25 años; (d) Desempleados a quienes se haya reconocido el abono de la prestación por desempleo en su modalidad de pago único, para su incorporación como socios trabajadores o de trabajo, siempre que el periodo de prestación por desempleo que tengan reconocido no sea inferior a 360 días si tienen 25 años o más y a 180 días si son menores de 25 años; (e) Mujeres desempleadas que se incorporen como socias trabajadoras o de trabajo en los 24 meses siguientes a la fecha del parto, adopción o acogimiento; (f) Desempleados con una discapacidad de grado de igual o superior al 33\%; y (g) Desempleados en situación de exclusión social pertenecientes a alguno de los colectivos contemplados en el Programa de Fomento del Empleo vigente en el momento de la incorporación como socio.

En relación a dichos colectivos y las modificaciones introducidas en la normativa autonómica, la mayoría ha optado por un criterio expansivo a efectos de incluir a más personas. Esta ampliación se ha hecho respecto a ciertos colectivos de personas desempleadas mencionadas o respecto del grupo genérico de personas desempleadas. Respecto del primero, cabe señalar como representativo la ampliación en Baleares, Aragón y Navarra del colectivo de menores de 25 años que no hayan tenido antes un primer empleo fijo para incluir a los menores de 30 años y sin excluir a aquellos que hubieran tenido un empleo fijo. Por su parte, la Comunidad Valenciana sólo amplía el margen de edad sin modificar la exigencia de no haber tenido empleo fijo. Respecto de la ampliación del grupo genérico, otras normativas autonómicas han optado por incluir a cualquier persona desempleada y, por lo tanto, cualquier persona que no cumpliera con ninguna de las circunstancias anteriormente listadas. Así, la norma de referencia en Cataluña sólo hace mención a personas desempleadas mientras que en Andalucía se incorpora una fórmula genérica para referirse a todas aquellas personas desempleadas "en quienes no concurran ninguna de las circunstancias previstas en los apartados anteriores" (Orden de 6 de junio de 2014). En el mismo sentido, Cantabria tan sólo exige como requisito estar inscritas en el Servicio Cántabro de Empleo con carácter previo y hasta la fecha de alta en la Seguridad Social y en los mismos términos se expresa la regulación en Castilla-La Mancha. Por su parte, Asturias opta por incluir como colectivo a las personas desempleadas mayores de 25 años y menores de 45 años, obteniendo por tanto los mismos efectos expansivos. Así mismo, la regulación en Aragón incluye como colectivo de posibles beneficiarias a las personas de entre 30 y 44 años, exigiendo como requisito contar al menos con 3 meses de inscripción como desempleado.

Dentro de las innovaciones más relevantes, podemos señalar el caso de la norma de referencia de Murcia, la cual prevé el colectivo de 'personas desempleadas' inscritas en cualquier oficina de colocación hasta la fecha de alta en el correspondiente régimen de la Seguridad Social, considerando igualmente sin empleo a "las personas que causen baja y alta en meses consecutivos en el Régimen Especial de trabajadores por cuenta propia o autónomos". Por su parte, en Galicia también se promueve la incorporación como personas socias trabajadoras o de trabajo a prueba de aquellas personas menores de 30 años que en el momento de la incorporación estén desempleadas e inscritas como demandantes de empleo. 
El segundo colectivo, recogido en la letra h) del artículo 3.1 de la Orden, es el conformado por las personas trabajadoras vinculadas a la empresa por contrato de trabajo de carácter temporal no superior a 24 meses, con una vigencia mínima de seis meses a la fecha de solicitud de la subvención. Lo primero que llama la atención es que la norma estatal no hace distinción, tampoco la autonómica, dentro de este colectivo como lo hace en relación con las personas desempleadas, cuando podría ser igualmente interesante fomentar el empleo de alguno de los colectivos mencionados también en el caso de estar empleados con contrato temporal. Así, las modificaciones introducidas por la normativa autonómica se ciñen a ampliar las personas beneficiarias al amparo de dicho precepto sin diferenciar colectivos dentro de este grupo. Por ejemplo, en el caso de Aragón, en dónde se suprime el límite superior de tiempo y se mantiene la antigüedad mínima de seis meses, y de Galicia, dónde se suprimen ambos límites de tiempo. En el caso de Cataluña, el colectivo de personas con vínculo contractual se amplía para acoger no sólo aquellos con contrato temporal sino también a aquellos con contrato fijo aún sin ser socios trabajadores o de trabajo. Otra modalidad contractual prevista en este caso en Baleares es aquélla de los trabajadores fijos discontinuos exigiéndose que el periodo de actividad inicialmente previsto fuera de seis meses como mínimo. Más allá de éstas, el caso de la reglamentación en Galicia destaca por la inclusión de las personas que en el momento de su incorporación sean socias a prueba de la cooperativa a la que se incorporan.

Fuera de esos colectivos que prevé la norma estatal y que las CCAA han adaptado a su territorio y contexto social podemos señalar otros colectivos incluidos en los efectos de dicha política de promoción de empleo estable y cuya incorporación responde a la propia actualización de la norma estatal. De esta forma, destaca la incorporación de las personas víctimas de la violencia de género (incluidas expresamente en Aragón y en Baleares) y las personas migrantes retornadas (en el caso de Galicia). Finalmente, como ejemplo de la actualidad de la política de promoción del empleo estable se quiere destacar aquí la regulación en Murcia que incluye a las personas inscritas en el Registro de la Garantía Juvenil.

\subsection{La calidad del empleo generado: criterios para la concesión de la subvención y las obligaciones asumidas por las entidades beneficiarias}

El empleo generado por las cooperativas y las sociedades laborales es, sin duda, uno de los rasgos más destacables a la hora de evaluar la capacidad de este tipo de sociedades a cumplir los fines que persiguen y es, por ello, que los poderes públicos deben fomentarlas. Ahora bien, lo que a continuación se pretende es estudiar cuáles son las características del empleo subvencionado que pueden relacionarse con un trabajo de mejor calidad. Aunque no es tarea fácil, la calidad del empleo generado se puede valorar en cuanto a los criterios utilizados por las administraciones para la resolución de la convocatoria de las subvenciones, por un lado, y las obligaciones asumidas por las cooperativas y las sociedades laborales beneficiarias de dichas subvenciones, por otro lado.

En cuanto a los criterios utilizados por las administraciones, lo primero que llama la atención es la ambigüedad de aquéllos previstos en la Orden estatal. Dicha norma recoge en el artículo 8.1 los criterios objetivos de valoración para resolver la 
concesión de la subvención serán: (a) el empleo generado; (b) el porcentaje de empleo de mujeres generado; (c) la contribución a la integración de colectivos en situación de exclusión social; (d) las actividades desarrolladas en el marco de los nuevos yacimientos de empleo definidos por la Unión Europea; (e) la incorporación de nuevas tecnologías de la información y las comunicaciones; (f) el desarrollo de proyectos de $\mathrm{I}+\mathrm{D}+\mathrm{i}$; (g) la incidencia en la creación y mantenimiento del empleo en el entorno geográfico en que se desarrolle la actuación; y (h) la contribución a la mejora del medio ambiente. Como se puede observar, los criterios mencionados adolecen de cierta imprecisión como, a modo ilustrativo, en lo relativo a cuáles deben considerarse los límites del entorno geográfico o la vigencia de los yacimientos de empleo determinados por la Comisión Europea hace más de dos décadas (Comunidades Europeas, 1993). Finalmente, la Orden prevé que todos los criterios tendrán la misma importancia "con independencia del orden en el que figuran recogidos".

La regulación existente a nivel autonómico es escasa, pero en algunos casos ha mejorado ostensiblemente los criterios recogidos en la Orden estatal ampliándolos así como precisando las previsiones sobre su aplicación. En cuanto a la ampliación de criterios, a modo de ejemplo, la regulación en Asturias introduce los 'novísimos yacimientos de empleo' consistentes en energías renovables (minihidráulica, biomasa, eólica, solar, térmica, geotermia), reforestación, deporte, agricultura ecológica, y organizaciones no gubernamentales. En el caso de Murcia, se incluye como factor valorable si la empresa redujo el índice de incidencia de accidentes laborales o se puntúa la realización de cursos de formación continua, directamente relacionado con el objeto social o actividad de la empresa y que organice ésta para sus socios trabajadores y/o trabajadores por cuenta ajena. En la normativa de la Comunidad Valenciana se introduce, por ejemplo, la implementación de políticas en materia de igualdad de oportunidades entre hombres y mujeres, la participación de mujeres en los órganos de dirección y administración de la entidad solicitante, la realización de balances, auditorías o informes, no preceptivos de carácter económico-social, medioambiental, o de sostenibilidad de la actividad de la empresa, o la utilización por la solicitante de productos procedentes del comercio justo, etc. Respecto de la precisión de los criterios para su aplicación, la regulación asturiana destaca por el grado de concreción de las variables, por ejemplo, en cuanto al criterio 'desarrollo de proyectos de Investigación, Desarrollo e Innovación' $(\mathrm{I}+\mathrm{D}+\mathrm{i})$ desglosando ésta en función del efectivo desarrollo de proyectos de $\mathrm{I}+\mathrm{D}+\mathrm{i}$ en las actividades de productividad industrial, diseño industrial y desarrollo de nuevos productos. En el caso de Baleares se incluye el grado de incidencia en la creación y el mantenimiento del empleo en el entorno geográfico, valorando si el índice de paro de la localidad donde se ubica el centro de trabajo en el que se produce la incorporación es superior al índice de paro en las Islas Baleares.

En cuanto a las obligaciones que deben cumplir las entidades de la economía social beneficiarias de las subvenciones, aquí se quiere destacar las siguientes por su impacto en la calidad del empleo. Por un lado, el art. 10.f) de la Orden estatal prevé que las cooperativas y sociedades laborales asumirán la obligación de mantener como socio trabajador o de trabajo a la persona o personas por cuya incorporación se concede la ayuda durante al menos tres años y, en el caso de que 
causara baja, aquellas entidades procederán a su sustitución por otra/s persona/s y por el período que reste hasta completar los tres años. Respecto de ello, algunas CCAA han rebajado el límite de dos años. Destaca el caso de Murcia la cual prevé que, para dar cumplida la obligación de mantener el puesto de trabajo durante tres años, el cómputo de los días cotizados por la persona que se conceda subvención sea, como mínimo, de mil noventa y cinco días (1.095 días).

Por otro lado, existen otras obligaciones que han incluido las CCAA en sus respectivas regulaciones y que se refieren a las condiciones de trabajo, especialmente en lo relativo al trabajo a tiempo parcial. La Orden estatal tan sólo prevé que, cuando la jornada de trabajo del socio trabajador o de trabajo incorporado sea a tiempo parcial, la cuantía de la ayuda será proporcional a la duración de su jornada. Respecto de esta posibilidad, en Aragón la normativa prevé la jornada de trabajo del socio trabajador incorporado será a tiempo completo o a tiempo parcial no inferior al $50 \%$ de la jornada ordinaria en la empresa, existiendo la obligación de mantener al menos durante dos años la jornada de trabajo del socio trabajador objeto de la subvención en un porcentaje como mínimo similar a aquel que se tuvo en cuenta para determinar el importe de la subvención. En Baleares se introduce que, en caso de incorporaciones de socios a tiempo parcial, se debe mantener ya no de forma similar sino como mínimo el porcentaje de jornada por el que se ha concedido la subvención, no pudiendo ser el porcentaje inferior al $50 \%$ de la jornada completa de un socio trabajador o de trabajo comparable. Por su parte, la regulación en Murcia obliga a que durante el período de los tres años las personas incorporadas como socias trabajadoras o de trabajo sólo podrán desarrollar una actividad a jornada completa.

Así pues, tras el análisis de los criterios establecidos como parámetros para valorar las solicitudes así como las obligaciones de las entidades beneficiarias después de la concesión, se puede afirmar que tanto unos como otros carecen de los suficientes elementos para potenciar el empleo de calidad.

En cuanto a los criterios, la regulación recogida en la Orden presenta dos problemas. En primer lugar, el problema consistente en una falta de coherencia entre las acciones subvencionables y los criterios aplicables en la valoración de las solicitudes. Así, la Orden enumera los criterios mencionados de aplicación tanto al procedimiento para resolver las subvenciones relativas a la incorporación de personas socias trabajadoras o de trabajo como a los procedimientos relativos a las subvenciones para la realización de inversiones y la prestación de asistencia técnica, también reguladas en la misma Orden. Es por ello que sea cuestionable que todos ellos deban ser aplicados indistintamente si las acciones subvencionables son claramente diferentes. En segundo lugar, los criterios que tienen una clara relación con la acción subvencionable de incorporación de personas socias al vincularse con el empleo generado -criterios de empleo generado, empleo femenino generado y contribución a la integración de colectivos en situación de exclusión social- se conciben desde una perspectiva cuantitativa que no cualitativa. En relación a ello, es destacable la conexión de esos criterios relativos al empleo generado con el requisito genérico recogido en el art. 3.1 por el cual la incorporación de las nuevas personas socias "deberá suponer un incremento del empleo respecto de la media de los 12 meses anteriores a la fecha de incorporación de los nuevos socios por los que se percibe la ayuda". En tanto que el efecto de este criterio es, al mismo 
tiempo, requisito para la concesión de la ayuda, sería más conveniente que, en lugar de adoptar dicha perspectiva cuantitativa, los criterios relativos al empleo se describieran en términos cualitativos valorando, por ejemplo, políticas de conciliación de la vida laboral y familiar. En cuanto a las obligaciones, a pesar de las modificaciones autonómicas que favorecen el empleo a tiempo completo o un mínimo de porcentaje del empleo a tiempo parcial, no existen otras obligaciones en relación a mejores prácticas laborales.

\section{Conclusiones: El impulso del empleo estable y de calidad a través de nuevos criterios y obligaciones}

Hace más de dos décadas, Garcia Marcos apuntaba a la directa relación entre la economía social y el fomento del empleo estable, y señalaba precisamente a las cooperativas de trabajo asociado y a las sociedades laborales como "instrumentos eficaces en la lucha contra el paro, tanto por la estabilidad y flexibilidad que ofrecen como por su contribución a la formación de una cultura empresarial" (García Marcos, 1996: 13-14). En este trabajo se ha pretendido aportar la perspectiva cualitativa al análisis del tipo de empleo generado a través de las políticas activas de empleo relacionadas con la incorporación de personas socias trabajadoras o de trabajo en cooperativas y sociedades laborales.

El método utilizado ha sido el análisis de la normativa autonómica en relación a las entidades que pueden ser beneficiarias, los colectivos cuya incorporación se subvenciona y, finalmente, los criterios establecidos en los procedimientos para la concesión de las subvenciones y las obligaciones asumidas por las entidades beneficiarias a las que se les otorga la ayuda. Tras el análisis de las normas autonómicas puede concluirse que, aunque difieren entre sí, la experiencia de la descentralización territorial es positiva. En primer lugar, es generalizada la ampliación de los colectivos cuya incorporación se subvenciona, lo cual facilita que un mayor número de personas socias puedan dar lugar a dicha subvención. En segundo lugar, el esfuerzo en algunas CCAA para precisar los criterios aplicables al procedimiento facilita no sólo que haya más transparencia sino además una orientación de la acción de las sociedades cooperativas y laborales que, en un futuro, quieran beneficiarse de dicha subvención. Y, en tercer lugar, es destacable la obligación recogida en algunas Comunidades Autónomas respecto de las limitaciones del uso de los contratos a tiempo parcial a fin de incrementar la relación laboral a tiempo completo.

Con todo, aquí se quiere llamar la atención de la necesidad de revisar los criterios recogidos en la Orden TAS/3501/2005 así como su incorporación en todas las normas de ámbito autonómico. Dicha revisión debería plantearse con el objetivo de, por un lado, reforzar las sostenibilidad social y medioambiental de las sociedades cooperativas y laborales y, por otro lado, promover aquellas sociedades que demuestren el cumplimiento de mejores condiciones laborales, haciéndose necesaria la distinción de los estándares exigidos a cooperativas y sociedades laborales.

Respecto de la sostenibilidad social, el concepto de yacimientos de empleo junto con los estudios de los observatorios ocupacionales deben ser parámetros 
para medir dicha sostenibilidad, pero también la economía social como alternativa de autoempleo digno. Un ejemplo es el uso de la fórmula cooperativa como alternativa frente a las nuevas formas de economía colaborativa a través de plataformas digitales, al tener puntos en común (Díaz Foncea, Marcuello Servós, y Monreal Garrido, 2016). En relación a ello, se ha demostrado que el sistema de trabajo alrededor de las plataformas digitales que operan en la economía colaborativa ocultan en ocasiones un alto grado de precariedad (De Rivera, Gordo López, y Cassidy, 2017). Así, el "platform cooperativism" propugnaría que la propiedad de las plataformas digitales que caracterizan ese tipo de economía sean de quienes proporcionan los recursos que las hacen funcionar (Alfonso Sánchez, 2016). En relación a la sostenibilidad medioambiental, Chacartegui Jávega (2018) ha destacado la interrelación entre las cooperativas y el empleo verde.

Respecto de establecer como criterios diferentes estándares sociales según sea la entidad beneficiaria una cooperativa o una sociedad laboral, es ineludible diferenciar dos baremos para cada tipo de sociedad atendiendo al diferente régimen jurídico de los trabajadores. Efectivamente, en tanto que el trabajo de los socios trabajadores de las cooperativas no se rige por la protección mínima aplicable a los socios trabajadores de las sociedades laborales, los estándares de valoración deben ser diferentes si realmente quieren los poderes públicos fomentar eficazmente un empleo de calidad. Así, en las CCAA en dónde el régimen jurídico del trabajo de los socios trabajadores no esté equiparado a las normas del derecho laboral común, pudiera tenerse como criterio favorable el hecho de que las entidades establezcan la máxima paridad con las garantías laborales del contrato por cuenta ajena (por ejemplo, aproximando los anticipos al Salario Mínimo Interprofesional) en sus estatutos y reglamentos de régimen interno, o bien otros parámetros como el menor porcentaje de socios trabajadores con contratos de duración determinada o la reducción del tiempo de periodo de prueba por debajo del límite legal. En el caso de socios trabajadores de sociedades laborales, al ser trabajadores asalariados, el baremo debiera tener en cuenta, por ejemplo, mejoras respecto del convenio colectivo de aplicación.

En definitiva, los poderes públicos deben cumplir con la Recomendación 193 OIT, la cual establece que las cooperativas "deben beneficiarse de condiciones conformes con la legislación y la práctica nacionales que no sean menos favorables que las que se concedan a otras formas de empresa y de organización social" (apartado 7.2). Así, Díaz Jiménez (2005: 405) afirma que de la Recomendación 193 se desprende "la aplicación, en régimen de igualdad, de la legislación laboral a todas las cooperativas y el reconocimiento de todos los derechos y garantías laborales, sin discriminación a todos sus socios trabajadores y de trabajo". Como ha dicho Bengoetxea (2015: 46) en relación a las cooperativas, "el empleo no se valora como coste productivo" sino que éste satisface el derecho al trabajo de los socios. Por ello, y pudiendo llegar a la misma conclusión en las sociedades laborales, los poderes públicos deben promover los mejores estándares laborales, teniendo como primera referencia el trabajo decente y sostenible. 


\section{Referencias Bibliográfícas}

Alfonso Sánchez, R. (2009) La legislación española sobre cooperativas y sociedades laborales: ¿una respuesta adecuada a las necesidades del sector?. CIRIEC-España, Revista de Economía Pública, Social y Cooperativa, № 20, pp. 31

Alfonso Sánchez, R. (2016) Economía colaborativa: un nuevo mercado para la economía social. CIRIEC-España, Revista de Economía Pública, Social y Cooperativa, No 88, pp. 231-258.

Arrieta Idiakez, F.J. (2014) Concreción de las entidades de la economía social. REVESCO. Revista de Estudios Cooperativos, Tercer Cuatrimestre, No. 116, p. 33-56. DOI: 10.5209/rev_REVE.2014.v116.45717.

Bengoetxea Alkorta, A. (2015) El empleo en las cooperativas de trabajo asociado: ¿derecho al trabajo o factor productivo?. Lan Harremanak, $\mathrm{N}^{\circ} 32$ (2015-I).

Bengoetxea Alkorta, A. (2016) Cooperativas. CIRIEC-España. Revista Jurídica No $29 / 2016$.

Calderón Milán, B. y Calderón Milán, M.J. (2012) La calidad del empleo de las entidades de la economía social en período de crisis. Ekonomiaz: Revista vasca de economía, $\mathrm{N}^{\mathrm{o}}$ 79, pp. 30-57.

Cavas Martínez, F. (2016a) Política de empleo como responsabilidad de los poderes públicos: el objetivo del pleno empleo. En VVAA La política y el derecho del empleo en la nueva sociedad del trabajo: Liber amicorum en honor de la profesora Rosa Quesada Segura. Consejo Andaluz de Relaciones Laborales. pp. 46-54.

Cavas Martínez, F. (2016b) La capitalización de las prestaciones por desempleo y el cese de actividad como medidas de fomento del emprendimiento social. Revista General de Derecho del Trabajo y de la Seguridad Social, $\mathrm{N}^{\circ} 43$

Chacartegui Jávega, C. (2018) Workers' Participation and Green Governance. Comparative Labor Law \& Policy Journal, Vol. 40, issue 1 - Fall 2018.

Chaves Ávila, R. y Savall Morera, T. (2013) La insuficiencia de las actuales políticas de fomento de cooperativas y sociedades laborales frente a la crisis en España. REVESCO. Revista de Estudios Cooperativos, Tercer Cuatrimestre, N ${ }^{\circ} 113$, pp. 61-91. DOI: 10.5209/rev_REVE.2014.v113.43383.

Clemente López, J., Díaz Foncea, M. y Marcuello Servos, M. (2009) Sociedades cooperativas y sociedades laborales en España: estudio de su contribución a la creación de empleo y al crecimiento económico. REVESCO. Revista de Estudios Cooperativos, Segundo Cuatrimestre, №. 98, pp. 35-69.

Colomer Viadel, A. (2017) Regulación constitucional de la participación de los trabajadores en la economía y en la empresa. Revista de Derecho Político, N 100 , pp. 831-848

Comisión Europea (2010) Europa 2020 Una estrategia para un crecimiento inteligente, sostenible e integrador. COM (2010) 2020 final.

Comunidades Europeas (1993) Crecimiento, competitividad, empleo. Retos y pistas para entrar en el siglo XXI. Libro Blanco. Luxemburgo: Oficina de Publicaciones Oficiales de las Comunidades Europeas.

Congreso de los Diputados (2010) Diario de Sesiones núm. 696 de 21 diciembre de 2010.

De Kostka Fernández Fernández, E. (2015) Comentario a la Ley 31/2015, de 9 de septiembre, por la que se modifica y actualiza la normativa en materia de autoempleo y se adoptan medidas de fomento y promoción del trabajo autónomo y de la Economía Social. Cooperativismo e economía social, No 38 , pp. 395-403.

Del Pino Domínguez (2016) Los principios de la economía social en la ley de sociedades laborales y participadas. CIRIEC-España. Revista Jurídica, № 29, pp.1-20. 
De Rivera, J., Gordo López, A. J. y Cassidy, P. R. (2017) La economía colaborativa en la era del capitalismo digital. Redes.com: Revista de Estudios para el desarrollo social de la Comunicación, $\mathrm{N}^{\circ}$ 15. pp. 20-31.

Díaz Foncea, M., Marcuello Servós, C. y Monreal Garrido, M. (2016) Economía social y economía colaborativa: Encaje y potencialidades. Economía industrial, No 402, pp. $27-$ 35.

Díaz Jiménez, M. (2005) La discriminación del trabajo cooperativizado. CIRIEC-España, Revista de Economía Pública, Social y Cooperativa, No 52, pp. 387-409.

Fajardo Garcia, I.G. (2012) El fomento de la "Economía Social" en la Legislación española. REVESCO. Revista de Estudios Cooperativos, Primer Cuatrimestre, № 107, pp. 58-97. DOI: $10.5209 / \mathrm{rev}$ REVE.2012.v107.38747.

García Jiménez, M. (2014) El desamparo del trabajo asociado por la legislación laboral o el limbo del trabajo cooperativizado. Revista General de Derecho del Trabajo y de la Seguridad Social, № 37. pp. 105-123.

García Marcos, C. (1996) La Economía Social como fomento de empleo. REVESCO. Revista de Estudios Cooperativos, № 62, pp. 13-16.

Montoya Melgar, A. (2004) El trabajo en la Constitución. Foro. Revista de Ciencias Jurídicas y Sociales, Nueva Época. No 0 . pp. 9-32

Nogueira Guastavino, M. (2018) Artículo 129: la participación de los interesados en la Seguridad Social y en la empresa. Comentario a la Constitución Española: 40 aniversario 1978-2018: Libro-homenaje a Luis López Guerra. Vol. 2. pp. 1799-1809

Oficina Internacional del Trabajo (2019) Trabajar para un futuro más prometedor Comisión Mundial sobre el Futuro del Trabajo. Ginebra: OIT.

Parlamento Europeo (2008) Resolución sobre economía social, de 19 de febrero de 2008 (2008/2250(INI))

Paniagua Zurera, M. y Jiménez Escobar, J. (2014) La necesidad de una legislación cooperativa adecuada: aspectos mercantiles, tributarios y de Derecho Comunitario. CIRIEC-España, Revista de Economía Pública, Social y Cooperativa, № 81, pp. 61-93.

Sala Ríos, M., Farré Perdiguer, M. y Torres Solé, T. (2014) Un análisis del comportamiento cíclico de las cooperativas y sociedades laborales españolas y de su relación con la actividad económica. REVESCO. Revista de Estudios Cooperativos, Segundo Cuatrimestre, No 115, pp. 7-29. DOI: 10.5209/rev_REVE.2014.v115.45279.

San Martín Mazzucconi, C. (2010) El enfoque preventivo de la lucha contra la exclusión social (II): la reducción de desigualdades a través de la explotación de nuevos yacimientos de empleo. La política de empleo como instrumento de inclusión social: un análisis jurídico. Madrid: Dykinson.

Trujillo Díez, I. J. (2002) Tutela judicial y efectiva de los socios trabajadores de cooperativas de producción: A propósito de la STC 86/2002, de 22 de abril. Derecho privado y Constitución, $\mathrm{N}^{\circ} 16$, pp. 393-430.

\section{Referencias legales}

Recomendación núm. 193 de la OIT sobre la promoción de las cooperativas.

Reglamento (UE) $N^{\circ}$ 1304/2013 del Parlamento Europeo y del Consejo, de 17 de diciembre de 2013, relativo al Fondo Social Europeo y por el que se deroga el Reglamento (CE) $\mathrm{N}^{\circ}$ 1081/2006 del Consejo. DOUE L347/ 470, 20 diciembre 2013.

Ley 2/2011, de 4 de marzo, de Economía Sostenible. BOE núm. 55, 5 marzo 2011.

Ley 5/2011, de 29 de marzo, de Economía Social. BOE núm. 76, 30 marzo 2011. 
Ley $31 / 2015$, de 9 de septiembre, por la que se modifica y actualiza la normativa en materia de autoempleo y se adoptan medidas de fomento y promoción del trabajo autónomo y de la Economía Social. BOE núm. 217, 10 septiembre 2015.

Real Decreto 1032/2017, de 15 de diciembre, por el que se aprueba la Estrategia Española de Activación para el Empleo 2017-2020.

Real Decreto Legislativo 3/2015, de 23 de octubre, por el que se aprueba el texto refundido de la Ley de Empleo. BOE núm. 255, 24 octubre 2015.

Decreto 22/2017, de 21 de marzo, por el que se regula la concesión directa de subvenciones en el marco del Programa de Promoción de Cooperativas y Sociedades Laborales en Castilla-La Mancha. Diario Oficial de Castilla-La Mancha. Núm. 61, 28 de marzo de 2017.

Decreto 89/2016, de 28 de junio, por el que se regula el Plan de ayudas para el fomento del empleo en la economía social, en el ámbito de la Comunidad Autónoma de Extremadura. Diario Oficial de Extremadura. Núm. 128, 5 de julio de 2016.

Orden TAS/3501/2005, de 7 de noviembre, por la que se establecen las bases reguladoras para la concesión de subvenciones para el fomento del empleo y mejora de la competitividad en las cooperativas y sociedades laborales.

Orden de 6 de junio de 2014, por la que se aprueban las bases reguladoras para la concesión de subvenciones del Programa de Apoyo a la Promoción y el Desarrollo de la Economía Social para el Empleo. Boletín Oficial de la Junta de Andalucía. Núm. 113.13 de junio 2014.

Orden EIE/607/2016, de 6 de junio, por la que se establecen las bases reguladoras para la concesión de subvenciones para la creación y consolidación del empleo y la mejora de la competitividad en cooperativas de trabajo asociado y sociedades laborales. Boletín Oficial de Aragón. Núm. 123, 28 junio 2016.

Orden HAC/13/2016, de 22 de abril de 2016, por la que se establecen las bases reguladoras de subvenciones destinadas al fomento del empleo y mejora de la competitividad en las cooperativas y sociedades laborales y a fi nanciar gastos de organización interna y funcionamiento de las asociaciones de cooperativas, de sociedades laborales y de asociaciones de trabajadores autónomos. Boletín oficial de Cantabria. Núm. 84, 3 de mayo de 2016.

Orden de 22 de diciembre de 2017, de la consejería de empleo, por la que se convocan las subvenciones para el año 2018 para la incorporación de socios trabajadores o de trabajo y la financiación de proyectos de inversión en cooperativas y sociedades laborales. Boletín Oficial de Castilla y León. № 42, 3 de marzo 2009.

Orden de 26 de diciembre de 2017 por la que se establecen las bases reguladoras para la concesión, en régimen de concurrencia no competitiva, de las subvenciones para el fomento del emprendimiento en economía social (Programa APROL-Economía Social), cofinanciadas parcialmente con cargo al Programa operativo del Fondo Social Europeo, y se convocan para el año 2018. Diario Oficial de Galicia. Núm. 18, 25 de enero de 2018.

Orden de 5 de octubre de 2017, de la Consejera de Economía, Empleo y Hacienda, por la que se establecen las disposiciones generales para la concesión de subvenciones para el fomento del empleo y mejora de la competitividad en las cooperativas y sociedades laborales en el ámbito de la Comunidad de Madrid. Boletín Oficial de la Comunidad de Madrid. Núm. 252, 23 de Octubre de 2017.

Orden de 2 de mayo de 2016 del titular de la Consejería de Desarrollo Económico, Turismo y Empleo, de bases reguladoras de subvenciones para programas de fomento de la 
Economía Social. Boletín Oficial de la Región de Murcia. Núm. 102, de 4 de mayo de 2016.

Orden de 4 de julio de 2018, de la Consejera de Trabajo y Justicia, por la que se convocan y regulan ayudas para la incorporación de personas socias a empresas de economía social. Boletín Oficial del País Vasco. Núm. 130, 6 de julio de 2018.

Ordre TSF/188/2016, de 27 de juny, per la qual s'aproven les bases reguladores que han de regir la convocatòria de subvencions per a la incorporació de persones sòcies treballadores o persones sòcies de treball en cooperatives i societats laborals. Diari Oficial de la Generalitat de Catalunya, núm. 7163, de 15 de julio 2016.

Resolució del conseller de Treball, Comerç i Indústria de 17 d'abril de 2018 per la qual s'aprova la convocatòria per concedir ajuts públics destinats a fomentar l'economia social per mitjà de la promoció de l'ocupació i la millora de la competitivitat de les cooperatives i les societats laborals. BOIB. Num. 73, 12 mayo 2005.

Resolución de 1 de agosto de 2013, de la Consejería de Economía y Empleo, por la que se aprueban las bases reguladoras para la concesión de ayudas a las cooperativas y sociedades laborales por la incorporación de socios trabajadores o socios de trabajo. Boletín Oficial del Principado de Asturias. Núm. 226, de 28 octubre 2013.

Resolución del presidente de la Agencia de Desarrollo Económico de La Rioja, de 26 de junio de 2000, por la que se aprueban las bases reguladoras de la concesión de ayudas al programa de fomento de la economía social. Boletín Oficial de La Rioja. Núm. 83.4 de julio de 2000.

Resolución de 19 de junio de 2018, de la Presidenta, por la que se aprueba la convocatoria para la concesión de subvenciones dirigidas a fomentar la incorporación de socios trabajadores o de trabajo a cooperativas y sociedades laborales de Canarias para el ejercicio 2018, cofinanciadas por el Fondo Social Europeo. Boletín Oficial de Canarias. Núm. 128, 4 de julio de 2018.

Resolución 682/2013, de 29 de abril, de la Directora Gerente del Servicio Navarro de Empleo, por la que se regula la concesión de subvenciones al fomento del empleo en las cooperativas de trabajo asociado y sociedades laborales de Navarra mediante la incorporación de socios en las mismas. Boletín Oficial de Navarra. Núm. 96, 22 de mayo de 2013.

Resolución de 1 de agosto de 2013, de la Consejería de Economía y Empleo, por la que se aprueban las bases reguladoras para la concesión de ayudas a las cooperativas y sociedades laborales por la incorporación de socios trabajadores o socios de trabajo). 